\title{
Double-Blind Controlled Trial of Vitamin E Effects on Serum Lipid Profile in Menopausal Women
}

This article was published in the following Dove Press journal:

Diabetes, Metabolic Syndrome and Obesity: Targets and Therapy

\author{
Parvaneh Rezasoltani $\mathbb{D}^{\prime}$ \\ Nahid Elliyoun' \\ Tahereh Ziaie ${ }^{2}$ \\ Abdolrasoul Sobhani ${ }^{3}$ \\ Ehsan Kazemnezhjad Leyli ${ }^{4}$ \\ Soudabeh Kazemi Aski $\mathbb{D}^{5}$ \\ 'Department of Midwifery, Social \\ Determinants of Health Research Center, \\ School of Nursing and Midwifery, Guilan \\ University of Medical Sciences, Rasht, \\ Iran; ${ }^{2}$ Department of Midwifery, School \\ of Nursing and Midwifery, Guilan \\ University of Medical Sciences, Rasht, \\ Iran; ${ }^{3}$ Department of Pharmacology, \\ Gastrointestinal and Liver Diseases \\ Research Center, School of Medicine, \\ Guilan University of Medical Sciences, \\ Rasht, Iran; ${ }^{4}$ Department of Biostatistics, \\ Road Trauma Research Center, School of \\ Nursing and Midwifery, Guilan University \\ of Medical Sciences, Rasht, Iran; \\ ${ }^{5}$ Department of Obstetrics \& \\ Gynecology, Reproductive Health \\ Research Center, School of Medicine, \\ Guilan University of Medical Sciences, \\ Rasht, Iran
}

\begin{abstract}
Background: Menopause is associated with changes in lipid profile and is a known risk factor for oxidative stress. Different therapeutical strategies have been used to control menopause complications. Vitamin E, an important anti-oxidant, can possibly affect lipid peroxidation in menopausal women. Thus, we aimed to evaluate the effect of vitamin $\mathrm{E}$ supplementation on the lipid profile of menopausal women.
\end{abstract}

Materials and Methods: This double-blind, placebo-controlled, randomized, cross-over, phase I/II trial study was designed in two 4-week intervention phases with an 8-day washout period in between. Eighty-three natural menopause women participated in the study. Randomized block allocation was used to divide women into group A $(n=41)$ and group B $(n=42)$. In phase I, one group received vitamin E capsule (400 IU/day) and another group received placebo capsule for 4 weeks. After an 8-day washout period, phase II was initiated for a period of 4 weeks, where the group that received vitamin $\mathrm{E}$ capsule was given placebo (E-P) and the group that received placebo was given vitamin E (P-E). Plasma lipid profile levels (LDL-C, HDL-C, TC, and TG) were assessed before and after intervention separately in each phase and in each group. Lipid profile was measured by enzymatic colorimetric method. Data were analyzed according to the intention-to-treat principle. All statistical analyses were performed using SPSS software.

Results: The analysis indicated no significant difference in plasma TC, LDL-C, HDL-C, and TG levels between P-E and E-P groups before intervention in phase I and II $(P>0.05)$. There was no significant difference in plasma lipid profile levels within the P-E and E-P groups before and after intervention in phase I and II. There was a significant difference in plasma TG within the E-P group before $[141.74 \pm 53.52,138.50$ (94-195)] and after [167.47 $\pm 71.32,170(108-202)]$ intervention in phase II $(P=0.010)$. There was no significant difference in terms of the mean changes in plasma lipid profile between the P-E and E-P groups in phase I and II $(P>0.05)$.

Conclusion: The study results revealed that vitamin E supplementation had no remarkable effect on the lipid profile in menopausal women.

Keywords: vitamin E, lipid profile, menopausal women

\section{Introduction}

Menopause is a transition from reproductive life to a permanent cessation of menstruation in the aging process. Menopause is due to loss of ovarian follicular function. Menopause is experienced by 1.5 million women each year. In industrialized countries, menopause occurs between the age of 45 and 55 years. ${ }^{1-3}$ Nowadays, menopause includes nearly one-third of a woman's lifetime. Menopause has various complications due to hormonal changes, including low estrogen, and increased luteinizing and follicular stimulating hormones.

\footnotetext{
Correspondence: Parvaneh Rezasoltani Department of Midwifery, School of Nursing \& Midwifery, Guilan University of Medical Sciences, Daneshjou Street, Shahid Beheshti Boulevared, Rasht, Guilan Province, 4I469-3984I, Iran

Tel $+98 \quad 1333555058$

Fax +98 1333550097

Email soltanip769@gmail.com
} 
Menopause complications include sexual dysfunction, urogenital atrophy, and central nervous system disorders.,

During menopause the ovaries cease to produce high estrogen levels, which are unveiled by metabolic changes and endothelial dysfunctions. ${ }^{5,6}$ The risks of developing hypertension, ischemic heart disease, myocardial infarction, and stroke increase following reduced estrogen production. ${ }^{7}$ Some studies revealed that menopause leads to changes in adipose tissue distribution, body mass index, and lipid metabolism. ${ }^{8}$ In this regard, studies reported that a decrease in estrogen level results in increased abdominal adiposity, which increments lipid profile, glucose, and insulin concentrations. ${ }^{9}$ It is accepted that menopause leads to changes in lipid profile by increasing total cholesterol (TC), low density lipoprotein (LDL-C), and triglyceride (TG) and by decreasing high density lipoprotein (HDL-C) level. ${ }^{10,11}$ These changes may cause cardiovascular disease (CVD). CVD is a leading cause of morbidity and mortality in menopausal women. ${ }^{12}$

Some studies showed positive effects for vitamin E supplementation, in fish oil, on reduction of total cholesterol and LDL-C in women with dyslipidemia during menopause transition. ${ }^{13}$ Other studies revealed the effectiveness of vitamin E on plasma lipid profile in oxidative stress-related diseases. ${ }^{14}$ It was previously reported that a combination of vitamin $\mathrm{C}$ and vitamin E reduced very low density lipoprotein (VLDL), LDL, and TG levels and increased HDL levels in postmenopausal women with breast cancer who were treated with tamoxifen. ${ }^{15}$ However, a recent study changed the ideas about this vitamin and showed that 2 months of vitamin E supplementation had no effect on lipid profile and adiponectin levels in obese adolescents. Therefore, there is inconsistency in the literature about the effects of vitamin $\mathrm{E}$ on lipid profile.

Based on the findings of the study by Peralta et al, vitamin $\mathrm{E}$ can be an alternative to estrogen. ${ }^{16}$ This hypothesis was evidenced by the findings of some studies that reported a reduction in vitamin $\mathrm{E}$ during menopause. ${ }^{17}$ According to the above-mentioned evidence, we proposed that vitamin $\mathrm{E}$ can affect the lipid profile in menopausal women. Accordingly, we aimed to assess the effect of vitamin E supplementation on plasma levels of HDL-C, LDL-C, TG, and TC in menopausal women.

\section{Materials and Methods}

\section{Study Design}

This study was conducted in the Alzahra Hospital, Guilan, Iran between March and November 2017. This double- blind, placebo-controlled, randomized, cross-over, phase $\mathrm{I} / \mathrm{II}$ trial was designed in two 4-week intervention phases separated with an 8-day washout period. Among 2000 menopausal women, 83 eligible volunteers who met the inclusion criteria participated in this study. Sample size was calculated according to formula number 1 below. We used randomized block allocation to ensure balance between the study groups during the recruitment period in this study. Therefore, the enrolled women were divided into group A $(n=41)$ and group B $(n=42)$. Vitamin E and placebo capsules were provided by the Daana Pharma Co. (Azerbaijan, Iran) according to the randomization algorithm. Participants and investigators were blinded to the intervention type. In phase I, one group was given vitamin E capsule (400 IU/day) and another group was given placebo capsule, which contained oral paraffin and had a similar shape and color to the vitamin E capsule, for 4 weeks. Then, subjects entered an 8-day washout period. The washout period length was determined based on the vitamin E half-life. Subsequently, phase II was initiated for a period of 4 weeks. In phase II the group that had received vitamin E capsule was given placebo (E-P) and the group that had received placebo was given vitamin $\mathrm{E}$ (P-E). The plasma lipid profile levels (LDL-C, HDL-C, TC, and TG) were assessed before and after each phase. The participants were followed-up weekly via telephone call for any possible side effects and to ensure adequate capsule intakes.

$$
n=\frac{\left(Z_{1}-\alpha / 2+Z_{1}-\beta\right)^{2}\left(S^{2}{ }_{1}+S^{2}{ }_{2}\right)}{2\left(\mu_{1}+\mu_{2}\right)^{2}}
$$

In this formula, $\mu_{1}$ and $\mu_{2}$ represent the mean for the first and second samples, respectively. $S_{1}$ and $S_{2}$ represent the variances for the first and second samples, respectively. $\alpha$ and $\beta$ represent type I and type II errors, respectively, and $Z$ represents confidence level.

\section{Inclusion/Exclusion Criteria}

Inclusion criteria were natural menopause, age between 45 and 60 years, at least a year elapse from the end of last menstruation, and body mass index (BMI) $<30 \mathrm{~kg} / \mathrm{m}^{2}$. The exclusion criteria were onset of any complications due to vitamin or placebo usage, irregular use of vitamin or placebo, vaginal bleeding, identifying any illness during the study process, failure to refer for blood sampling, history of diabetes, hypertension, dyslipidemia, cardiovascular, kidney, or liver diseases, as well as cancer, having 
any sports activities, consumption of medications like hormonal replacement therapy (HRT) or supplementary vitamins in the past 6 months, consumption of alcohol and smoking, and $\mathrm{BMI} \geq 30$.

\section{Ethical and Dissemination}

The Ethics Committee and Institutional/Ethical Review Board of the Guilan University of Medical Sciences, Rasht, Iran approved this study (IR.GUMS. REC.1395.189). All study procedures were performed in accordance with the last edition of the Declaration of Helsinki guidelines. Eligible participants provided written informed consent. The research was registered in the Iranian Registry of Clinical Trials on 20 February, 2017 (Registration code: IRCT2016063028717N1).

\section{Data Collection}

\section{Lipid Profile Measurement}

Blood samples were collected after 12-14 hours of overnight fasting before and after each study phase. Measurement of the lipid profile, including LDL-C, HDL-C, TC, and TG, was performed by enzymatic colorimetric method using a Pars Azmoon kit (Tehran, Iran). Briefly, the non-hemolyzed blood samples were centrifuged. Measurements were conducted by an experienced laboratory technician according to the kit manufacturer instructions using a BT 2000 Plus automatic analyzer (Biotecnica, Italy).

\section{Statistical Analysis}

Statistical analysis was performed using the statistical package for social sciences (SPSS) software (IBM Corp., Armonk, NY, USA) version 22.0. The KolmogorovSmirnov and Shapiro-Wilk tests as well as the Stem-andLeaf Plot were used to assess normal distribution of data. The data distribution was not normal, so data were analyzed with an independent $t$-test, and the Wilcoxon Signed Rank and Mann-Whitney $U$ non-parametric tests. The study outcomes (lipid profile) were compared within and between placebo-vitamin E (P-E) and vitamin E-placebo (E-P) groups in each phase separately. Furthermore, the overall lipid profile was analyzed between vitamin $\mathrm{E}$ and placebo groups by gathering and comparing the data from the groups in both phases. Data were analyzed based on both the per-protocol (PP) and intention-to-treat (ITT) analyses. Sample sizes for ITT analysis were 41 participants in the P-E group and 42 participants in the E-P group. The study results were presented as mean $\pm \mathrm{SD}$, median, and percentile $(25,75) . P<0.05$ was considered statistically significant.

\section{Results}

A total of 83 women participated in the study. At the end of phase II, 13 dropped out of the study. There was no significant difference between $\mathrm{P}-\mathrm{E}$ and $\mathrm{E}-\mathrm{P}$ groups in terms of baseline characteristics, including age (mean = $52, \mathrm{SD}=4.32$ vs mean $=52.82, \mathrm{SD}=4.39$, respectively), weight $($ mean $=69.44, \mathrm{SD}=6.74$ vs mean $=69.86, \mathrm{SD}=$ 6.63 , respectively), height $($ mean $=160.54, \mathrm{SD}=5.58$ vs mean $=159.59, \mathrm{SD}=5.53$, respectively $), \mathrm{BMI}($ mean $=$ $26.92, \mathrm{SD}=2.41$ vs mean $=27.45, \mathrm{SD}=2.11$, respectively), age of the spouse (mean $=57.18, \mathrm{SD}=5.94 \mathrm{vs}$ mean $=58.18, \mathrm{SD}=7.91$, respectively), age of menopause $($ mean $=52, \mathrm{SD}=4.30$ vs mean $=52.80, \mathrm{SD}=4.30$, respectively), and the time elapsed since the last menstrual period $($ mean $=54.66, \mathrm{SD}=52.30$ vs mean $=64.68, \mathrm{SD}=$ 47.66, respectively). Therefore, the study comprised homogeneous samples. Moreover, PP (Table 1) and ITT analyses showed no significant difference between $\mathrm{P}-\mathrm{E}$ and E-P groups in plasma lipid profile, including TC, LDL-C, HDL-C, and TG, before intervention in phase I and II.

According to the results of PP (Table 2) and ITT analyses, there was no significant difference in plasma TC, LDL-C, HDL-C, and TG levels within the P-E group and in plasma TC, LDL-C, and HDL-C levels within the E-P group before and after intervention in phase I and II. According to the results of PP (Table 2) and ITT analyses, the plasma levels of TG was significantly different within the E-P group before [141.74 \pm $53.52,138.50(94-195)]$ and after $[167.47 \pm 71.32,170$ (108-202)] intervention in phase II.

PP analysis (Table 3) and ITT analysis found no significant difference for the mean changes of plasma $\mathrm{TC}$, LDL-C, HDL-C, and TG levels between the P-E and E-P groups in phase I and II. Although the mean changes of plasma TG levels in phase II had a borderline significant trend between the P-E group $(-6.30 \pm 60.31)$ and the E-P group $(-25.74 \pm 55.18)$ in ITT analysis $(P=0.057)$.

Overall, in this study no significant difference was observed in the mean and median of plasma TC, LDL-C, HDL-C, and TG levels in the placebo group $(n=83)$ compared to the vitamin E group $(n=83)$ before and after intervention based on both PP (Table 4) and ITT analyses. Based on Table 4 the median plasma TC [205 (178-237.50)], LDL-C [120.80 (105.80-151)], and HDL-C [47 (40-55)] levels in the vitamin E group increased compared to the 


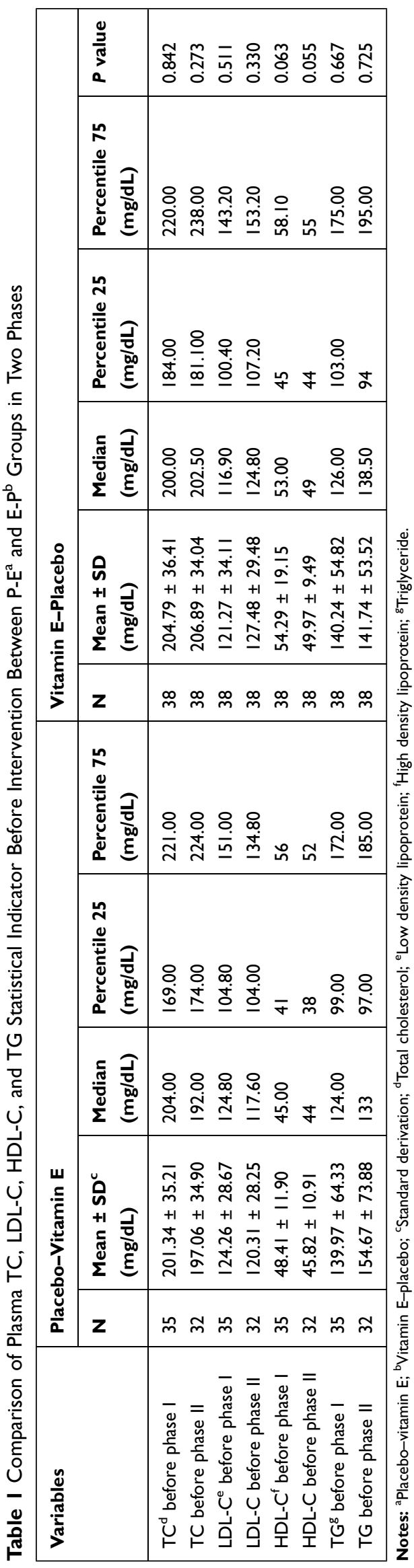

placebo group [201 (170-230); 117.20 (95.80-152.80); 46 (39-53)]. The median of plasma TG [134 (99-179)] levels in the vitamin $\mathrm{E}$ group decreased compared to the placebo group [142 (103-191)], although these changes were not significant. The median change in plasma TC $[-4.50(-20$ to 12$)$ ], LDL-C [ -4.60 ( -15.4 to 12$)]$, and TG [ -3.50 ( -32 to 24)] was lower in the vitamin $E$ group compared to the placebo group [1 (-13 to 19); 4.40 (-14.40 to 24$) ;-3$ ( -49 to 18)]. The median change in plasma HDL-C in the vitamin E group [1 ( -3 to 6$)]$ was higher compared to the placebo group [0 (-3 to 3$)$ ], which was not significant.

\section{Discussion}

In the present clinical trial, 83 menopause women between the age of 45 and 60 years were evaluated. We aimed to evaluate the possible effect of vitamin E supplementation on plasma lipid levels. Overall, the study results revealed that vitamin E supplementation did not show any significant advantage in terms of lowering plasma lipid profile levels in comparison to placebo.

In the current study, the median plasma TG level was $138.50 \mathrm{mg} / \mathrm{dL}$ (94-195) before placebo intervention in the E-P group in phase II, which increased to $170 \mathrm{mg} / \mathrm{dL}$ (108-202) after placebo intervention. This change was statistically significant. Furthermore, median levels of other factors including TC, LDL-C, and HDL-C were not significantly different in both phases within P-E and E-P groups. In this regard, Aryono et al in 2019, showed that 2 -month administration of $400 \mathrm{IU} /$ day of vitamin $\mathrm{E}$ in obese adolescents did not have a considerable impact on lipid profiles and adiponectin levels. Some studies concluded that vitamin E supplementation could be effective in the pharmacotherapy of menopausal symptoms especially in situations that HRT side effects were not tolerable. ${ }^{18}$ In a study by Wang et al, the effect of different doses of vitamin $\mathrm{E}$ on plasma lipid status was evaluated in women with metabolic syndrome. Their results indicated a significant decrease in TC levels in patients. ${ }^{14}$ Recently in a randomized, double-blind, placebo-controlled trial, the effect of 3-month omega-3 supplementation with or without a vitamin $\mathrm{E}$ component on oxidative biomarkers and lipid profiles was investigated in menopause women with T2DM. They declared that omega-3 plus vitamin E significantly reduced TC and LDL-C. ${ }^{19}$ In the present study, the median TC level decreased after placebo intervention in phase I and increased after vitamin E consumption in phase II. However, these changes were not statistically significant. Vitamin E is a major lipid soluble anti-oxidant 


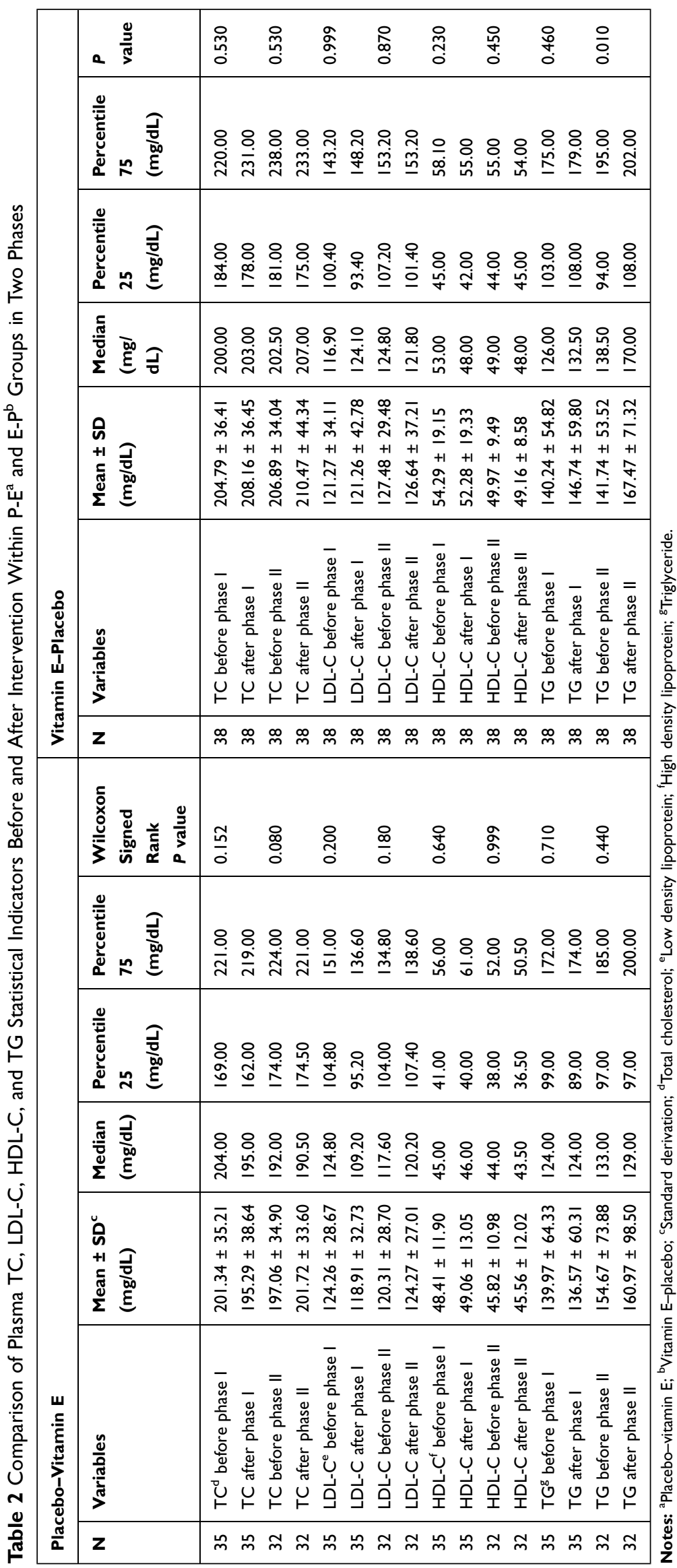



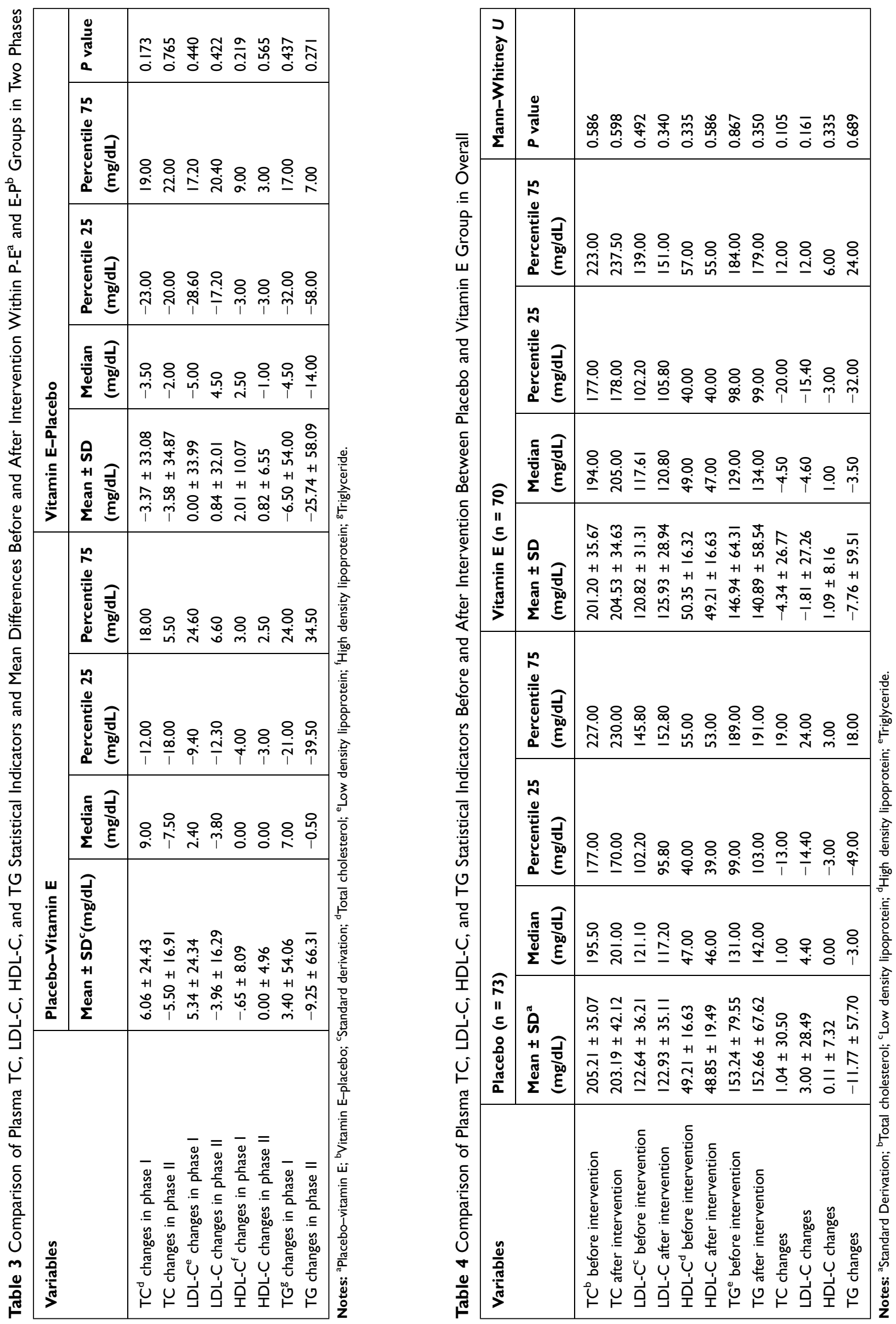
that is carried by cholesterol-rich lipoproteins in the plasma. Therefore, the slightly higher serum TC levels after vitamin intervention in the current study may be related to the higher plasma level of vitamin $\mathrm{E}$ at the intervention time. Moreover, to the best of knowledge, no similar study has yet been done; therefore, it is possible that if the sample size had been larger, the results would have been different.

In the current study, no significant difference was seen in mean changes of lipid profile before and after intervention in two groups. Khabaz et al evaluated the effect of daily $800 \mathrm{mg} / \mathrm{dL}$ vitamin $\mathrm{E}$ administration in diabetic patients (over 30 years old) for 3 months. In contrast to the findings of the current study, their results indicated that vitamin E consumption did not have any significant effect on plasma lipid profile. ${ }^{20}$ Miquel et al studied the relationship between hot flushes, OS, and the risk of CVD, and declared that vitamin anti-oxidants such as vitamin $\mathrm{E}$ supplemented with vitamin $\mathrm{C}$ reduced LDL and LDL-C oxidation, that lead to prevention of atherosclerosis in postmenopausal women. ${ }^{21}$ Studies revealed that vitamin E inhibits lipid peroxidation mediated by free radicals but not enzymatic oxidation by lipoxygenase and cyclooxygenase, which are important in the pathogenesis of diseases such as atherosclerosis. ${ }^{22,23}$ A meta-analysis of randomized controlled trials evaluated the efficacy of vitamin and anti-oxidant supplements in prevention of CVD and stated that unplanned and high doses of vitamin $\mathrm{E}$ administration had no beneficial effect in preventing CVD. $^{24}$ In line with the findings of the current study, Lucas et al assessed the dose-dependent effects of vitamin E supplementation on lipid parameters in ovariectomized rats and declared that vitamin E had no significant effect on serum lipid parameters. ${ }^{25}$

There is poor evidence from randomized controlled trials about the effectiveness of complementary therapies including vitamin $\mathrm{E}$ consumption on menopausal symptoms. The different and sometimes controversial findings could be due to the differences in study design, type of administration, dosage, long-term or short-term consumption of vitamin E, and treatment endpoints. A review study by Rees on complementary therapies in postmenopausal women reported that some researchers claim that the antioxidant supplements reduce mortality, while others pretense that the use of anti-oxidants, including vitamin $\mathrm{E}$ and A, led to more deaths. ${ }^{26}$ Therefore, it seems that more studies are needed in order to achieve more conclusive results.

\section{Conclusion}

Based on the current study findings, vitamin E supplementation had no considerable effect on the plasma lipid profile in menopausal women. Evaluation of the effects of vitamin $\mathrm{E}$ on lipid profile is a new aspect of vitamin E related research. Thus, further studies are needed in this regard.

\section{Data Sharing Statement}

The datasets used and/or analyzed in the current study are available from the corresponding author upon reasonable request.

\section{Acknowledgments}

This study was done with financial support provided by Vice Chancellor of Research, Guilan University of Medical Sciences, Rasht, Iran (95060612).

\section{Disclosure}

The authors report no conflicts of interest in this work.

\section{References}

1. Santoro N, Epperson CN, Mathews SB. Menopausal symptoms and their management. Endocrinol Metab Clin North Am. 2015;44 (3):497-515. doi:10.1016/j.ecl.2015.05.001

2. Burger HG, Hale GE, Robertson DM, Dennerstein L. A review of hormonal changes during the menopausal transition: focus on findings from the Melbourne women's midlife health project. Hum Reprod Update. 2007;13(6):559-565. doi:10.1093/humupd/dmm020

3. Kooti W, Mansouri E, Ghasemiboroon M, Harizi M, Amirzargar A. Protective effects of celery (Apium Graveolens) on testis and cauda epididymal spermatozoa in rat. Iran J Reprod Med. 2014;12(5):365-366.

4. Jehan S, Masters-Isarilov A, Salifu I, et al. Sleep disorders in postmenopausal women. J Sleep Disord Ther. 2015;4(5):1000212.

5. Stachowiak G, Pertyński T, Pertyńska-Marczewska M. Metabolic disorders in menopause. Prz Menopauzalny. 2015;14(1):59-64.

6. Maturana MA, Irigoyen MC, Spritzer PM. Menopause, estrogens, and endothelial dysfunction: current concepts. Clinics. 2007;62:7786. doi:10.1590/S1807-59322007000100012

7. Harvey RE, Coffman KE, Miller VM. Women-specific factors to consider in risk, diagnosis and treatment of cardiovascular disease. Womens Health. 2015;11(2):239-257. doi:10.2217/WHE.14.64

8. Lizcano F, Guzmán G. Estrogen deficiency and the origin of obesity during menopause. Biomed Res Int. 2014;2014:757461. doi:10.1155/ 2014/757461

9. Mauvais-Jarvis F, Clegg DJ, Hevener AL. The role of estrogens in control of energy balance and glucose homeostasis. Endocr Rev. 2013;34(3):309-338.

10. Bade G, Shah S, Nahar P, Vaidya S. Effect of menopause on lipid profile in relation to body mass index. Chron Young Sci. 2014;5 (1):20-24

11. Derby CA, Crawford SL, Pasternak RC, Sowers M, Sternfeld B, Matthews KA. Lipid changes during the menopause transition in relation to age and weight: the study of women's health across the nation. Am J Epidemiol. 2009;169(11):1352-1361. doi:10.1093/aje/kwp043

12. Gorodeski GI. Update on cardiovascular disease in post-menopausal women. Best Pract Res Clin Obstet Gynaecol. 2002;16(3):329-355. doi:10.1053/beog.2002.0282 
13. Alves Luzia L, Mendes Aldrighi J, Teixeira Damasceno NR, et al. Fish oil and vitamin e change lipid profiles and anti-ldl-antibodies in two different ethnic groups of women transitioning through menopause. Nutricion hospitalaria. 2015;32(1):165-174.

14. Wang Q, Sun Y, Ma A, Li Y, Han X, Liang H. Effects of vitamin E on plasma lipid status and oxidative stress in Chinese women with metabolic syndrome. Int J Vitam Nutr Res. 2010;80(3):178-187. doi:10.1024/0300-9831/a000015

15. Babu JR, Sundravel S, Arumugam G, Renuka R, Deepa N, Sachdanandam P. Salubrious effect of vitamin $\mathrm{C}$ and vitamin $\mathrm{E}$ on tamoxifen-treated women in breast cancer with reference to plasma lipid and lipoprotein levels. Cancer Lett. 2000;151(1):1-5. doi:10.1016/S0304-3835(99)00340-7

16. Peralta EA, Brewer AT, Louis S, Dunnington GL. Vitamin E increases biomarkers of estrogen stimulation when taken with tamoxifen. J Surg Res. 2009;153(1):143-147. doi:10.1016/j.jss.2008.03.030

17. Mata-Granados JM, Cuenca-Acebedo R, Luque de Castro MD, Quesada Gomez JM. Lower vitamin E serum levels are associated with osteoporosis in early postmenopausal women: a cross-sectional study. $J$ Bone Miner Metab. 2013;31(4):455-460. doi:10.1007/s00774-013-0432-2

18. Hendarto A, Alhadar AK, Sjarif DR. The Effect of Vitamin E Supplementation on Lipid Profiles and Adiponectin Levels in Obese Adolescents: A Randomized Controlled Trial. Acta Medica Indonesiana. 2019 Apr 1;51(2):110-6.

19. Mahmoodi MR, Kimiagar M, Mehrabi Y. The effects of omega-3 plus vitamin $\mathrm{E}$ and zinc plus vitamin $\mathrm{C}$ supplementation on cardiovascular risk markers in postmenopausal women with type 2 diabetes. Ther Adv Endocrinol Metab. 2014;5(4):67-76. doi:10.1177/ 2042018814548028
20. Khabaz M, Rashidi M, Kaseb F, Afkhami-Ardekani M. Effect of vitamin $\mathrm{E}$ on blood glucose, lipid profile and blood pressure in type 2 diabetic patients. Iran J Diabetes Obes. 2009;1(1):11-15.

21. Miquel J, Ramirez-Boscá A, Soler A, et al. Increase with age of serum lipid peroxides: implications for the prevention of atherosclerosis. Mech Ageing Dev. 1998;100(1):17-24. doi:10.1016/ S0047-6374(97)00118-8

22. Phaniendra A, Jestadi DB, Periyasamy L. Free radicals: properties, sources, targets, and their implication in various diseases. Indian $J$ Clin Biochem. 2015;30(1):11-26.

23. Mansouri E, Kooti W, Bazvand M, et al. The effect of hydro-alcoholic extract of foeniculum vulgare mill on leukocytes and hematological tests in male rats. Jundishapur J Nat Pharm Prod. 2015;10(1): e18396. doi:10.17795/jjnpp-18396

24. Myung SK, Ju W, Cho B, et al. Efficacy of vitamin and antioxidant supplements in prevention of cardiovascular disease: systematic review and meta-analysis of randomised controlled trials. $B M J$ (Clinical Res Ed). 2013;346:f10.

25. Lucas EA, Chen TY, Chai SC, et al. Effect of vitamin E on lipid parameters in ovariectomized rats. $J$ Med Food. 2006;9(1):77-83. doi:10.1089/jmf.2006.9.77

26. Rees M. Alternative treatments for the menopause. Best Pract Res Clin Obstet Gynaecol. 2009;23(1):151-161. doi:10.1016/j. bpobgyn.2008.10.006

\section{Publish your work in this journal}

Diabetes, Metabolic Syndrome and Obesity: Targets and Therapy is an international, peer-reviewed open-access journal committed to the rapid publication of the latest laboratory and clinical findings in the fields of diabetes, metabolic syndrome and obesity research. Original research, review, case reports, hypothesis formation, expert opinion and commentaries are all considered for publication. The manuscript management system is completely online and includes a very quick and fair peer-review system, which is all easy to use. Visit http://www.dovepress.com/testimonials.php to read real quotes from published authors. 